\title{
Development of Dissolution Tests for the Quality Control of Complementary/Alternate and Traditional Medicines: Application to African Potato Products
}

\author{
Vipin Devi Prasad Nair ${ }^{1}$ and Isadore Kanfer \\ Division of Pharmaceutics, Faculty of Pharmacy, Rhodes University, Grahamstown, South Africa. \\ Received, January 3, 2008; Revised, March 24, 2008; Accepted, June 3, 2008; Published, June 5, 2008.
}

ABSTRACT - PURPOSE. Unlike orthodox
medicines, specific guidelines for dissolution
testing of complementary/alternate (CAMs) and
traditional medicines (TMs) have not been
developed nor is dissolution testing a requirement
for the quality control of such products. In this
report, the dissolution of African Potato (AP)
products, an African traditional medicine (ATM)
which has been ingested by man for a diversity of
ailments, has been investigated. A norlignan
glycoside namely hypoxoside and a sterol, $\beta-$
sitosterol (BSS) are purported to be the most
important phytochemicals in marketed products
of AP. Dissolution testing of AP products
containing labelled content of sterols and those
containing only hypoxoside is proposed whereby
BSS and hypoxoside are monitored as markers for
the release of the contents of the abovementioned
products, respectively. METHODS. The FDA
dissolution guidance for industry was used to
study the best dissolution condition for several
formulations of AP. Buffers in the range of pH
1.2 to 7.5 were used to investigate the dissolution
of AP products containing hypoxoside as a
marker compound. Similarly, biorelevant dissolution media such as fasted state simulation fluid (FaSSIF) and fed state simulation fluid (FeSSIF) at different $\mathrm{pH}$ were used to investigate the release of BSS in AP formulations labelled to contain sterols which exhibited poor water solubility. RESULTS. Dissolution testing of AP products containing hypoxoside, conducted at $\mathrm{pH}$ 1.2 using USP Apparatus 1 indicated that more than $75 \%$ of hypoxoside was released within $1 \mathrm{hr}$. Dissolution testing of products containing sterols, conducted in FeSSIF at a pH of 5.0 resulted in a release of at least $75 \%$ of BSS after $1 \mathrm{hr}$ for all but one of the products tested. CONCLUSIONS. Dissolution testing conditions have been developed for AP products containing two different marker compounds where one of the components, hypoxoside, is water soluble, whereas another component, BSS is poorly water soluble. This necessitated the use of different dissolution media and $\mathrm{pHs}$ in order to monitor the respective release of hypoxoside and BSS from AP products.

The results of this study indicate the necessity and possibility of developing appropriate dissolution testing procedures for use in the quality control of CAMs/TMs.

\section{INTRODUCTION}

The increasing popularity and use of complementary/alternate (CAMS) and traditional medicines (TMs) has stimulated the need to develop appropriate guidelines and methods for the quality control of such medicines and their pharmaceutical dosage forms. Amongst African traditional medicines (ATMs) African Potato (AP), Hypoxis hemerocallidea, also known as Hypoxis rooperi, which belongs to the family Hypoxidaceae, is purported as possibly being the best-known medicinal plant by many South Africans (1). Apart from its perceived nutritional value, it is of great medical interest (2) and extracts of the corms have been ingested by man for a diversity of ailments (3) including for the treatment of urinary diseases (4), prostate hypertrophy and internal cancer (5). Furthermore, it gained increased prominence as an alternative medicine for nutritional use in the daily diet of HIV/AIDS patients as a result of strong recommendations by the South African Minister of Health (6). AP contains hypoxoside which is a norlignan di-glucoside present in corms of Hypoxis plants (4) (Figure 1).

\footnotetext{
Corresponding Author: Professor Isadore (Izzy) Kanfer, Faculty of Pharmacy, Rhodes University Grahamstown, South Africa, E-mail: I.Kanfer@ru.ac.za

${ }^{1}$ Current Address: Arkansas State University, Jonesboro, Arkansas, USA.
} 
This glycoside possesses low toxicity and AP is used as a food (7), whereas its aglycone, rooperol, is purported to possess antiphologistic, bacterostatic and bactericidal properties (8). The vast pharmacological and clinical reports (9-11) have led to the registration of several patents (1214) and the commercialization of the extract of AP as commercially available formulations with various therapeutic claims. Traditionally, AP is cut into cubes or shredded and boiled for 20 minutes before the decoction is orally consumed. It has also been claimed that sterols and sterolins present in AP are responsible for its medicinal properties, but this has yet to be scientifically proven. Sterols such as $\beta$-sitosterol (BSS), stigmasterol (STG) and stanols like stigmastanol (STN) have secured an important place in the realm of health supplements with extensive scientific support for their prophylactic and therapeutic use for various physical ailments such as atherosclerosis $(15,16)$, benign prostatic cancer (17) and colon cancer (18, 19). Many reports of their medicinal properties are based on in vitro data or unrealistic high in vivo doses, making the therapeutic application of these compounds highly questionable (20-22).

Several formulations of AP are promoted for their sterol content (23) especially, BSS, which is shown in Figure 2.
Currently, no monograph or QC methods have been described for such commercial formulations containing AP. Although assay methods for use in the quality control (QC) of AP products have been developed and described (24-26), a further important QC criterion, namely, dissolution testing still needs to be addressed. The maintenance of the release properties required to effect consistent absorption from the gastrointestinal tract (GIT) is considered as an important feature and valuable quality control (QC) parameter. The quality, safety and efficacy of formulations can only be assured when all the appropriate quality control methods, including dissolution testing, have been applied.

Guidelines for dissolution testing of oral dosage forms for orthodox medicines have been developed by the food and drug administration (FDA) (27). However, the application of such guidelines to $\mathrm{CAMs} / \mathrm{TMs}$ has been complicated by the lack of data to confirm the utility/activity of components in such medicines as well as for many herbal extracts and products. In a few countries such as Germany, the quality requirements for dosage forms containing single active entities are also applicable to herbal medical products (HMPs) whereas in the USA, HMPs are generally considered as nutritional supplements.

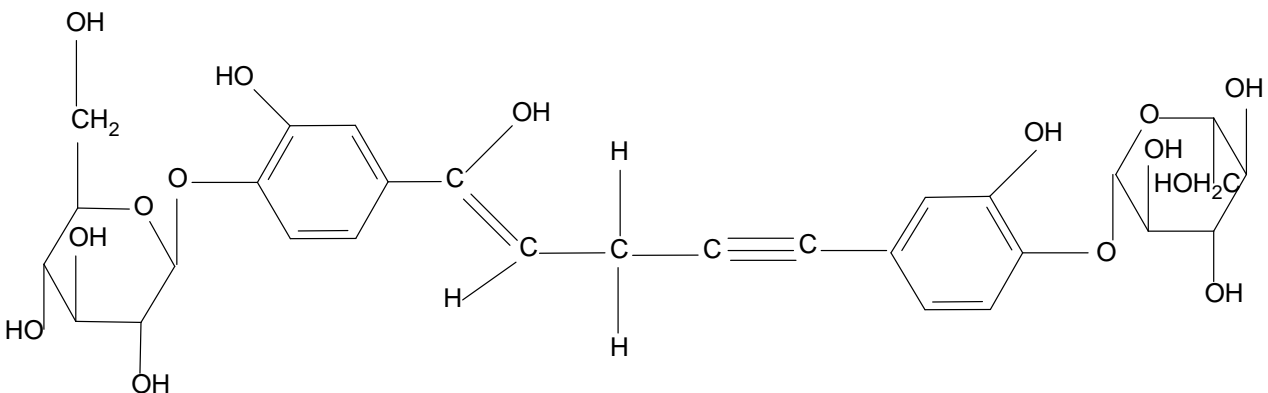

Figure 1. Chemical structure of hypoxoside

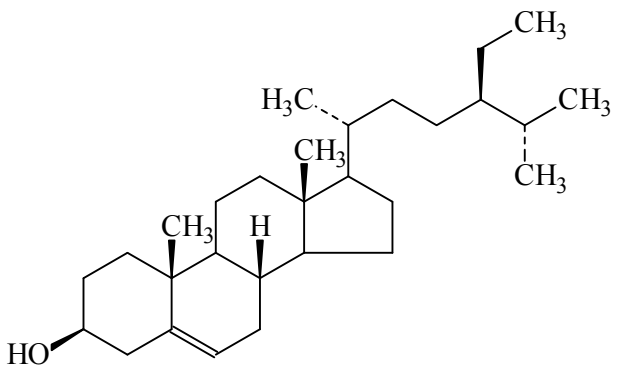

Figure 2. Chemical structure of $\beta$-sitosterol (BSS) 
CAMs and ATMs are generally quite complex, consisting of multiple components that may be active individually or in combination. The "actives" in CAMs and ATMs are usually defined to be the whole herbal preparation, e.g. the extract in its entirety. The USP (28) suggests that for botanicals, compliance with dissolution testing is performed by testing six or more dosage units individually in each vessel and measuring one or more index marker compounds or the extract specified in the individual monograph.

In Europe, the European Agency for the Evaluation of Medicinal Products (EMEA) proposes three categories of HMPs based on the characterization of their active components (29). Category A consists of standardised extracts for which an active moiety/moieties has/have been definitively identified. Standardisation of such products may be achieved by adjusting the level of actives by the addition of extracts or inert excipients that have higher or lower levels of the desired active compounds; examples: silymarin, aescin and sennoside (30). Category B consists of quantified extracts for which the active ingredient(s) has/have not been clearly identified which are known to contribute to the pharmacological or synergistic activity. Standardisation of these products may be achieved by blending batches of either raw botanical material or herbal preparations of higher or lower quality; however, the addition of inert excipients is not performed; examples: ginsenosides, procyanidins, flavonoids and hyperforin (30). Category $\mathrm{C}$ consists of unknown active ingredients for which no individual active ingredients have been identified, but have a traditional place in the therapy of certain diseases. Here, chemical compounds which may not contribute to any pharmacological activity are selected as markers for Good Manufacturing Practice (GMP); examples: extracts of valerian and echinaceae (30). The EMEA exempts dissolution tests for HMPs falling under the categories $\mathrm{B}$ and $\mathrm{C}$ if the product is being formulated as an immediate release. The dissolution test could be substituted with a disintegration test if the active ingredient is known (category A) to be soluble in aqueous solutions at $\mathrm{pH}$ values typical of the GIT (31).

For the purpose of evaluation of pharmaceutical and biopharmaceutical quality, dissolution testing can thus be readily applied to those products for which the components that contribute to the activity have been identified. Hence, since dissolution testing is seen as appropriate for HMPs under category A, dissolution testing requirements for AP products, a category A HMP, would therefore apply.

To-date there have been no reported methods to evaluate the in vitro dissolution profile of AP products based on their sterols (BSS and STG) or stanols (STN) content. There are also no methods or data on the in vitro dissolution profile of AP products based on hypoxoside content.

The FDA dissolution guidance for industry (27) recommends the use of buffers in the range of $\mathrm{pH} 1.2$ to 7.5 for initial dissolution method development. Several other types of dissolution media such as milk, simulated gastric fluid (SGF), simulated intestinal fluid (SIF), fasted state simulation fluid (FaSSIF) and fed state simulation fluid (FeSSIF) (33), have been proposed for the purpose of possible prediction of in vivo performance of some drugs. FaSSIF has usually been used at a $\mathrm{pH}$ of 6.5 because this particular $\mathrm{pH}$ was intended to simulate intestinal $\mathrm{pH}$ and provide useful information to predict in vivo dissolution in the fasted state. FeSSIF is slightly different from FaSSIF in that that medium contains an acetate buffer instead of phosphate buffer in order to simulate the conditions of the upper small intestine. The preferred $\mathrm{pH} 5.0$ of FeSSIF is considered to simulate the fed state of the intestine in the human body (33).

During the analysis of some commercially available AP products it was found that BSS was the major component present in some of those AP products when compared to the content of other sterols and stanols (24). This sterol was consequently chosen as the marker for the dissolution testing of AP products with labelled content of BSS. In addition, hypoxoside was chosen as a marker for products which were assayed (25) and found to contain quantifiable amounts of this norlignan component.

\section{MATERIALS AND METHODS}

\section{INSTRUMENTATION}

A Hanson SR8 PLUS Autoplus ${ }^{\mathrm{TM}}$, Multifill ${ }^{\mathrm{TM}}$ and Maximiser syringe fraction collector (Chatsworth, CA, USA) were used for the dissolution studies. A Mettler dual range electronic balance, Type AE 163 (Mettler Instruments AG, Griefensee, Zurich, Switzerland) was used for weighing the standards and samples. A Crison GLP21 pH Meter (Crison, Barcelona, Spain) was used to measure and adjust 
relevant $\mathrm{pH}$ values. Analyses of the phytochemicals were carried out using the equipment and validated analytical methods previously reported $(24,25)$.

Hypoxoside in the formulations was assayed and the dissolution samples at different time intervals were analyzed on an Alliance 2690 HPLC system (Waters Corporation, Milford, MA, USA) equipped with a 2996 photodiode array (PDA) detector, a degasser, a column heater and auto sampler. A Luna $\mathrm{C}_{18}(2)(5 \mu \mathrm{m}, 150 \times 4.6 \mathrm{~mm}$ ID) column (Phenomenex, Torrance, CA, USA) was used at $23 \pm 2{ }^{\circ} \mathrm{C}$. A mobile phase consisting of acetonitrile: water in isocratic mode (20: 80, $\mathrm{v} / \mathrm{v}$ ) was used at a flow rate of $1 \mathrm{~mL} / \mathrm{min}$ using a detection wavelength of $260 \mathrm{~nm}(24,26)$.

The samples for BSS were analyzed on the same Alliance 2690 HPLC system but using a Phenomenex Luna $C_{8}$ Column $(5 \mu \mathrm{m}, 50 \times 4.6$ $\mathrm{mm}$ ID). The chromatographic elution was accomplished isocratically with methanol-water (95: 5, v/v) at a flow rate of $1 \mathrm{ml} / \mathrm{min}$. The temperature was maintained at $23 \pm 1{ }^{\circ} \mathrm{C}$ and the injection volume was $10 \mu \mathrm{l}$. After detection by $\mathrm{UV}$, the chromatographic column effluent was subjected to quantitative analysis detection by ELSD (Alltech 2000, Alltech associates, Inc., Deerfield, USA). Nebulisation of the effluent in the ELSD was provided by a stream of pressurized air $(0.7 \mathrm{~L} / \mathrm{min})$ and the nebulised effluent was evaporated at $100{ }^{\circ} \mathrm{C}$. The detector was set at a gain of 16 , with output interfaced, via a SATIN box, to a Waters Empower ${ }^{\circledR}$ Chromatographic Manager (24).

\section{REAGENTS AND CHEMICALS}

Methanol (HPLC Grade) was purchased from Romil Ltd (The Source, Waterbeach, Cambridge, UK). Stigmasterol (95\%), stigmastanol (95\%) and $\beta$-sitosterol (97\%) were purchased from Sigma (St. Louis, MO, USA) and cholesterol from Croda Chemicals Ltd. (North Humberside, UK). Sodium taurocholate ( $>98 \%)$ and egg-phosphatidylcholine (99.1\%) were purchased from Sigma (Missouri, USA). Potassium dihydrogen phosphate, sodium hydroxide pellets, sodium dihydrogen phosphate and potassium chloride, were of analytical grade obtained from Rochelle Chemicals (Port Elizabeth, South Africa). Acetic acid of analytical grade was purchased from BDL Chemicals Ltd, (Poole, UK). Water was obtained from a Milli-Q system (Millipore, Bedford, MA, USA) and all samples were filtered using Durapore (PVDF) filters purchased from the same source.

Seven formulations (Products A, B, C, D, $\mathrm{E}, \mathrm{F}$ and $\mathrm{G})$ containing AP were purchased from a local retail pharmacy in Grahamstown, South Africa. Six of the products (Products A, B, D, E, $F$ and $G$ ) were capsules and only Product $C$ was in tablet form. All the above products were individually analysed to determine their content of hypoxoside using a validated analytical method (25). Only products A, B and C (no label claims made for BSS content) showed the presence of hypoxoside and were thus used to monitor the dissolution of hypoxoside.

Products, D, E, F and G were purported to be fortified with additional quantities of sterols and sterolins. The presence of BSS was determined using a validated HPLC-ELSD method (24). Hence these formulations were subsequently used to develop dissolution profiles for the BSS content. The information on different dosage forms and their assay value are shown in Table 1 .

\section{METHODS}

\section{Dissolution conditions}

Dissolution tests for Product C (tablet dosage form) were performed using the USP-2 (Paddle) apparatus (28) whereas the USP-1 (Basket) apparatus was used for all the capsule dosage forms (Products A, B, D, E, F and G). Each of the vessels was filled with $900 \mathrm{ml}$ of the appropriate dissolution medium and the temperature of the vessel contents were maintained at $37 \pm 0.5^{\circ} \mathrm{C}$. The rotation speed of the paddle as well as the basket was set at 100 rotations per min (rpm).Volume adjustments of the media were made by replacement of the withdrawn sample volume with fresh buffer at the same $\mathrm{pH}$ stored in a reservoir at the same temperature.

\section{Analyses of the dissolution samples}

Samples of about $2 \mathrm{ml}$ were withdrawn from the dissolution vessels through $0.2 \mu \mathrm{m}$ in-line filters at various time intervals and $0.2 \mathrm{ml}$ of relevant internal standard was added to $1.8 \mathrm{ml}$ of each sample. Sulphamerazine $(10 \mu \mathrm{g} / \mathrm{ml})$ was used as internal standard for the hypoxoside measurements and cholesterol $(50 \mu \mathrm{g} / \mathrm{ml})$ was used for the samples analyzed for BSS. 
Table 1. List of AP Products

\begin{tabular}{|c|c|c|c|c|}
\hline Sample Name & Average Weight of Product & Label Claim/Unit & $\begin{array}{c}\text { Hypoxoside } \\
\text { (mg/dosage unit) }\end{array}$ & \%RSD \\
\hline Product - A & $0.590 \mathrm{~g}$ & African potato $70 \mathrm{mg}$ & $12.42 \pm 0.6$ & 6.18 \\
\hline Product - B & $0.660 \mathrm{~g}$ & Hypoxis rooperi $250 \mathrm{mg}$ & $21.67 \pm 0.4$ & 3.22 \\
\hline Product - C & $1.214 \mathrm{~g}$ & AP powder $275 \mathrm{mg}$ & $18.75 \pm 0.9$ & 5.17 \\
\hline Sample Name & Average Weight of Product & Label Claim/Unit & $\begin{array}{c}\beta \text {-Sitosterol } \\
\text { (mg/dosage unit) }\end{array}$ & $\%$ RSD \\
\hline Product - D & $0.551 \mathrm{~g}$ & $\begin{array}{l}232 \mathrm{mg} \text { Hypoxis hemerocallidea } \\
+40 \mathrm{mg} \text { sterols and sterolins }\end{array}$ & $50.02 \pm 0.4$ & 2.78 \\
\hline Product - E & $0.638 \mathrm{~g}$ & $\begin{array}{l}15 \mathrm{mg} \text { Hypoxis extract }+30 \mathrm{mg} \\
\text { sterol and sterolin extracts }\end{array}$ & $28.12 \pm 0.8$ & 3.92 \\
\hline Product - F & $0.516 \mathrm{~g}$ & $25 \mathrm{mg}$ plant sterols and sterolins & $13.51 \pm 0.7$ & 5.67 \\
\hline Product - G & $0.278 \mathrm{~g}$ & $\begin{array}{l}\text { Plant sterols } 20 \mathrm{mg} \text { and plant } \\
\text { sterolins } 0.2 \mathrm{mg}\end{array}$ & $21.18 \pm 0.6$ & 4.28 \\
\hline
\end{tabular}

Adjustments in the calculations were done to compensate for dilutions of dissolution media as a result of volume replacement following sample withdrawal and the cumulative amount (mg) of drug released at different time intervals was calculated. These values were then converted to $\%$ cumulative drug release with respect to the assay value of the respective formulations and the relevant dissolution profiles were constructed.

\section{In Vitro Dissolution Method Development}

The prescribed dissolution conditions according to the USP were used to monitor the release of the relevant components of AP. However, in view of the fact that BSS is relatively insoluble under aqueous conditions, the usual conditions for dissolution testing were considered inappropriate to monitor the release of this component from AP products. Hence, biorelevant dissolution media such as FaSSIF and FeSSIF containing lecithin as emulgent and bile salts as solubilizer were chosen as an option to monitor the release of BSS from AP products (34).

\section{Products containing hypoxoside}

The USAs FDA dissolution guidance for industry (27) recommends the use of buffered dissolution media in the range of $\mathrm{pH} 1.2$ to 7.5. However, since hypoxoside is relatively unstable at higher
$\mathrm{pH}$ values, dissolution media at two $\mathrm{pH}$ values (1.2 and 4.5) were used for these studies. The dissolution medium at $\mathrm{pH} 1.2$ was prepared from hydrochloric acid $(100 \mathrm{mM})$, whereas the dissolution medium at $\mathrm{pH} 4.5$ was prepared with $100 \mathrm{mM}$ phosphoric acid and the $\mathrm{pH}$ adjusted with sodium hydroxide pellets. Product B was chosen to establish the optimum $\mathrm{pH}$ for dissolution. This product was chosen since it contained a high content of hypoxoside (21.67 $\mathrm{mg}$ /dosage unit). AP tablets were added to each of six dissolution vessels containing $900 \mathrm{ml}$ of the respective dissolution medium. Samples were withdrawn at intervals of $0.5,1.0,2.0,4.0,6.0$, 8.0 and $12.0 \mathrm{hr}$ and were analyzed using the validated HPLC method. The results of the study revealed similar profiles for Product B in both $\mathrm{pH} 1.2$ and 4.5 where $\sim 99 \%$ hypoxoside was released after $4 \mathrm{hr}$ at $\mathrm{pH} 1.2$ and a slightly lower value at $\mathrm{pH} 4.5$ (Figure 3 ). From the results of these studies, dissolution media at either $\mathrm{pH} 1.2$ or 4.5 appeared to be suitable for use in the dissolution testing of hypoxoside.

\section{Products containing $\beta$-sitosterol}

BSS is relatively insoluble in aqueous media and samples of AP product containing BSS were exposed to 4 different dissolution media ( $\mathrm{pH} 1.2$, 4.5, 6.8 and 7.8) and analyzed after $12 \mathrm{hr}$. 


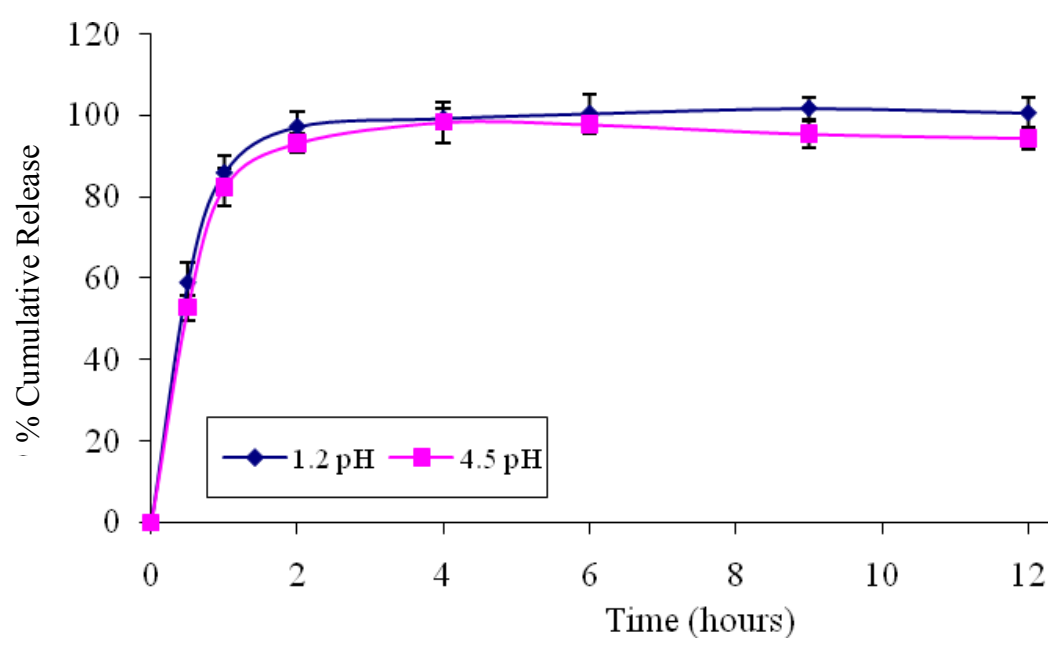

Figure 3. Dissolution of product-B for hypoxoside at $\mathrm{pH} 1.2$ and 4.5

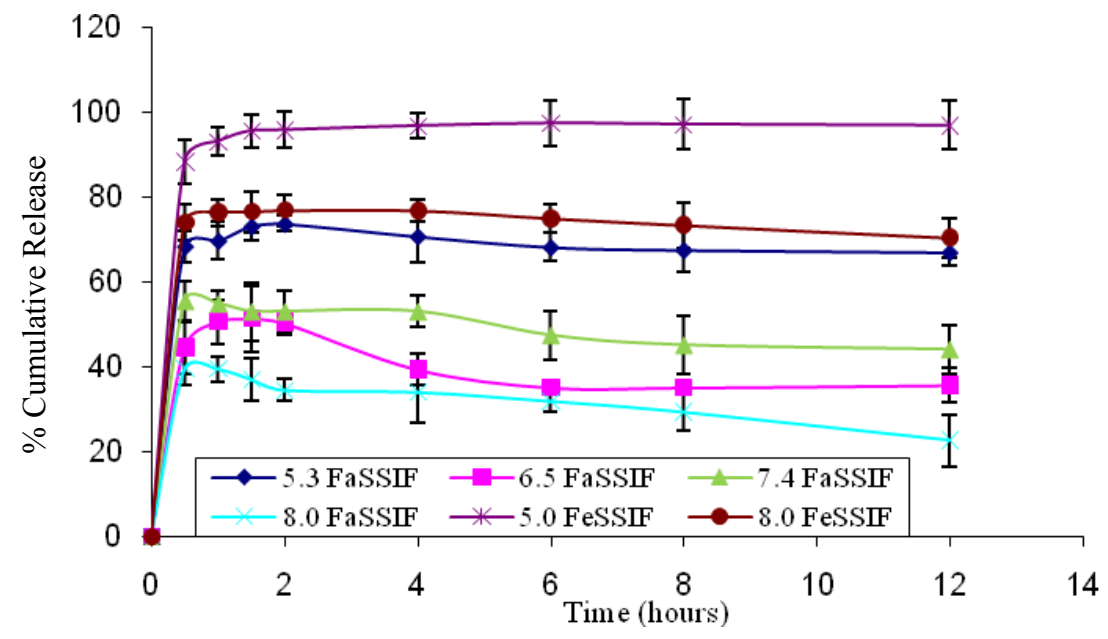

Figure 4. Dissolution of product-D for $\beta$-sitosterol in FaSSIF and FeSSIF at various pHs

BSS was not detected in any of the samples, thereby confirming the compounds aqueous insolubility. As a result, alternative dissolution media were considered for the dissolution testing of BSS. FaSSIF is a dissolution medium which has been proposed by Dressman et al (33) as a medium which represents the fasted state in the proximal small intestine of humans. Although FaSSIF is generally used at a $\mathrm{pH}$ of 6.5 , four different pHs, 5.3, 6.5, 7.4 and 8.0 were used for these dissolution studies since the current objective was not to attempt to correlate in vitroin vivo results but rather to establish an appropriate discriminating dissolution medium for use with a relatively insoluble compound such as $\mathrm{BSS}$, for the QC of products containing this sterol.

Product D was used and samples were withdrawn at the previously indicated intervals and following analysis by HPLC, BSS dissolution was found to be higher at $\mathrm{pH} 5.3$ using FaSSIF $(\sim 70 \%)$ when compared to the higher pH's (Figure 4).

FeSSIF was subsequently investigated to determine whether it would provide dissolution conditions to effect a higher degree of dissolution. Whereas the use of FeSSIF is usually conducted at a preferred $\mathrm{pH}$ of $5.0, \mathrm{a} \mathrm{pH}$ of 8.0 was also 
investigated. Analysis of the samples of Product $\mathrm{D}$ indicated that $\sim 88$ and $\sim 93 \%$ of BSS was released in the first $30 \mathrm{~min}$ and one $\mathrm{hr}$ respectively at $\mathrm{pH} 5.0$ compared to the release at the higher $\mathrm{pH}$ of 8.0 , where $\sim 73$ and $\sim 78 \%$ of BSS was released at $30 \mathrm{~min}$ and one $\mathrm{hr}$ respectively. It is thus apparent that the inclusion of bile salts and lecithin facilitate the dissolution of BSS in addition to the effects of $\mathrm{pH}$. Justification for the inclusion of bile salts and lecithin can be gleaned from previously reported data where the bioavailability of stigmastanol, which has similar physicochemical properties to BSS, increased in the presence of lecithin by $34 \%$ following a $300 \mathrm{mg}$ dose (35).

It is therefore seen that in view of the fact that the usual aqueous dissolution media are not applicable to determine the dissolution of BSS, the alternate dissolution medium of FeSSIF at $\mathrm{pH}$ 5.0 was an acceptable choice. Hence, this medium was selected to study the in vitro release profile of different products containing BSS.

\section{Dissolution of products containing hypoxoside}

The dissolution of the different products containing AP was conducted at $\mathrm{pH} 1.2$ under the previously mentioned conditions using six dissolution vessels for each of products A, B and C.

\section{Dissolution of products containing $\beta$-sitosterol}

The dissolution of the different products containing BSS was conducted at $\mathrm{pH} 5.0$ under the previously mentioned conditions using FeSSIF as dissolution medium. Each product was placed in six dissolution vessels and samples were withdrawn at the previously described intervals.

\section{RESULTS}

\section{Dissolution profiles of products containing hypoxoside}

The samples of products $\mathrm{A}, \mathrm{B}$ and $\mathrm{C}$ were analyzed at $\mathrm{pH} 1.2$ using USP Apparatus 1 and their \% cumulative release was calculated (Figure 5). All the products under this condition released more than $75 \%$ hypoxoside within one $\mathrm{hr}$. However, the release of hypoxoside from product A reached a maximum of $\sim 80 \%$ and did not increase further as seen by the plateau on that product's profile. Product $C$ (tablet) showed the highest percentage release after one $\mathrm{hr}(\sim 95.1)$ compared to product A (capsule), whereas product B (capsule) showed the same release of hypoxoside as was previously shown during the development studies.

\section{Dissolution profiles of products containing $\beta$ - sitosterol}

The dissolution profiles in FeSSIF medium $(\mathrm{pH}$ 5.0) of products D, E, F and G containing BSS are shown in Figure 6. All the products except product $\mathrm{E}$ released at least $75 \%$ of BSS after one hr. Only $\sim 47 \%$ BSS was released from product E after one hr but increased to $\sim 86 \%$ at $12 \mathrm{hr}$. Release of BSS from product $\mathrm{G}$ was greater than any of the other products $(\sim 100 \%$ at $12 \mathrm{hr})$ even though there appeared to be a slight lag in dissolution between $0.5-2 \mathrm{hr}$. Dissolution of BSS from products $\mathrm{D}$ and $\mathrm{F}$ reached a plateau after two $\mathrm{hr}(>80 \%$ after $1 \mathrm{hr})$ where dissolution was seen to be $>90 \%$.

\section{DISCUSSION}

The release profiles of most of the products studied, apart from the release of BSS from product $\mathrm{E}$ and the incomplete release of hypoxoside from product A imply that in general, the dissolution data support the quality of the respective products and also may provide useful information with respect to absorption, in particular, BSS following administration of products $\mathrm{D}, \mathrm{F}$ and $\mathrm{G}$.

Over the past few decades, in vitro dissolution testing has been considered to be an important tool to assess and control variables associated with formulation which may alter the release characteristics of the active moiety from a pharmaceutical dosage form. Notwithstanding, in vitro dissolution testing is one of the most important and useful test methods for assuring product quality, which can aid the selection and development of prototype formulations and help optimize the formulation to provide a desirable drug release profile. This feature also helps to assure product quality and batch-to-batch consistency. Furthermore, it is also a valuable tool to assess drug product stability and shelf-life since the dissolution characteristics of products may change over time. Considering the above advantages, dissolution testing can be used as an important component in the QC of CAMs/ATMs since it provides information on the rate and amount of active compound (s) which is/are released over a specific period of time. 


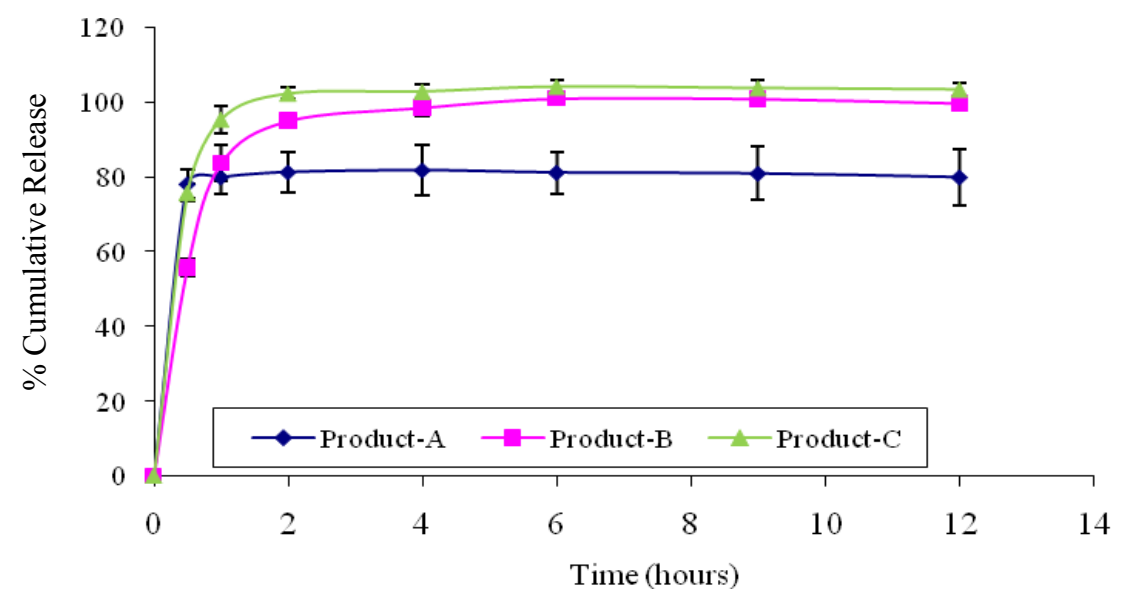

Figure 5. Dissolution profiles of different products containing hypoxoside in phosphate buffer (pH 1.2)

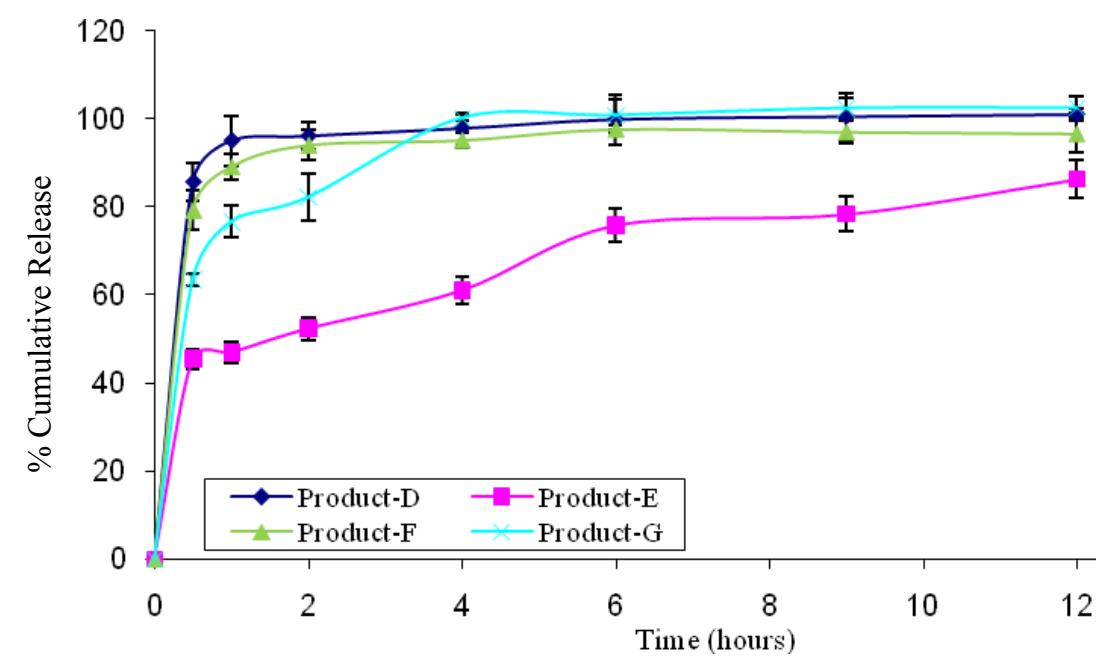

Figure 6. Dissolution profiles of different products containing $\beta$-sitosterol in FeSSIF (pH 5.0)

In vitro dissolution for medicinal products is considered pertinent, both from the QC point of view and also from bioavailability considerations. Furthermore, considering the above features, and more particularly using biorelevant dissolution media such as FaSSIF \&/or FeSSIF, in vitro dissolution can be a useful predictor of bioavailability and subsequent clinical performance of products provided that an acceptable in vitro-in vivo correlation (IVIVC) has been established.

\section{CONCLUSIONS}

Dissolution testing conditions have been developed for AP products containing two different marker compounds where one of the components, hypoxoside, is water soluble, whereas another component, BSS is poorly water soluble. This necessitated the use of different dissolution media and $\mathrm{pHs}$ in order to monitor the respective release of hypoxoside and BSS from AP products. 
Since AP products would likely fall under the EMEA Category A (30), dissolution testing of $\mathrm{AP}$ products and other $\mathrm{CAM} / \mathrm{TM}$ products falling within the abovementioned classification should thus be a necessary QC procedure for use in the quality control of CAMs/TMs.

\section{ACKNOWLEDGEMENT}

This work was supported by research grants from the South African Medical Research Council (MRC) and the National Research Foundation (NRF).

\section{REFERENCES}

[1]. S E. Drewes and F Khan. The African Potato (Hypoxis hemerocallidea) a chemical-historical perspective. S A J Sci, 100:425-430, 2004.

[2]. M. Nicoletti, C. Galeffi, I. Messana and G B. Marini-Bettolo. Hypoxidaceae: Medicinal uses and the nor-lignan constituents. J Ethnopharmacol, 36:95-101, 1992.

[3]. S E. Drewes and R W. Liebenberg. Pentenediphenyl-diglucoside containing compound. 4,652,636, U.S., 1987.

[4]. G B. Marini-Bettolo, M. Patamia, M. Nicoletti, C. Galeffi and I. Messana. Research on African medicinal plants-II: Hypoxoside, a new glycoside of uncommon structure from hypoxis obtusa busch. Tetrahed, 38:1683-1687, 1982.

[5]. P. Vinesi, M. Serafini, M. Nicoletti, L. Spano and P. Betto. Plant regeneration and hypoxoside content in Hypoxis obtusa. J Nat Prod, 53:196199, 1990.

[6]. Southern African Development Community (SADC) Meeting on nutrition and HIV/AIDS, Johannesburg, South Africa, 2002.

[7]. S E. Drewes, A J. Hall, R A. Learmonth and U J. Upfold. Isolation of hypoxoside from Hypoxis rooperi and synthesis of (E)-1, 5-bis (3', 4'-dimethoxy phenyl) pent-4-en-1-yne. Phytochem, 23:1313-1316, 1984.

[8]. S E. Drewes and R W. Liebenberg, 2 120650, Great Britain, U.K., 1982.

[9]. B J. Smit, C F. Albrecht, R W. Liebenberg, P B. Kruger, M. Freestone, and L. Gouws. A phase I trial of hypoxoside as an oral prodrug for cancer therapy - absence of toxicity. S Afr Med J, 85:865-870, 1995.

[10]. C F. Albrecht, P B. Kruger, B J. Smit, M. Freestone, L. Gouws and R. Miller. The pharmacokinetic behavior of hypoxoside taken orally by patients with lung cancer in a phase I trial. S Afr Med J, 85:861-865, 1995.

[11]. C F. Albrecht, E J. Theron and P B. Kruger. Morphological characterization of the cellgrowth inhibitory activity of rooperol and pharmacokinetic aspects of hypoxoside as an oral prodrug for cancer therapy. S Afr Med J, 85:853-860, 1995.

[12]. K H. Pegel. Active plant extracts of hypoxidaceae and their use. 4,198,401. U.S., 1980.

[13]. G B. Marini-Bettolo, J. Msonthi, C. Galeffi, I. Messana, M. Nicoletti, 49561 A/83, Italy, 1983.

[14]. S E. Drewes and R W. Liebenberg. 0092226, Europe, 1983.

[15]. T A. Miettinen, P. Puska, H. Gylling and E. Vartiainen. Reduction of serum cholesterol with sitostanol-ester margarine in a mildly hypercholesterolemic population. New Engl $\mathbf{J}$ Med, 333:1308-1312, 1995.

[16]. H F J. Hendriks, J A. Westerate, T. van Vliet and G W. Meijer. Spreads enriched with three different levels of vegetable oil sterols and the degree of cholesterol lowering in normocholesterolaemic and mildly hypercholesterolaemic subjects. Eur J Clin Nutr, 53:319-327, 1999.

[17]. H. Schilcher and H. Schneider. Role of Sterols in the treatment and prophylaxis of benign prostatic cancer. Urologe B, 30:62, 1990.

[18]. A V. Rao and S A. Janezic. The role of dietary phytosterols in colon carcinogenesis. Nutr Cancer, 18:43-52, 1992.

[19]. A V. Rao and R. Koratkar, Antinutrients and Phytochemicals in Food in F. Shahidi (ed), American Chemical Society, Washington DC., U.S.A., pp 313-324, 1997.

[20]. R J. Marles and N R. Farnsworth. Plants as sources of antidiabetic agents. Econ Med Plant Res, 6:149-187, 1994.

[21]. R J. Marles and N R. Farnsworth. Antidiabetic plants and their active constituents. Phytomed, 2:137-189, 1995.

[22]. K B. Hicks and R A. Moreau. Phytosterols and phytostanols: functional food cholesterol busters. Food Technol, 55:63-67, 2001.

[23]. K H. Pegel and R W. Liebenberg. Extraction of sterolins from plant material. 3,933,789, U.S., 1976.

[24]. V D P. Nair, I. Kanfer and J. Hoogmartens. Determination of stigmasterol, $\beta$-sitosterol and stigmastanol in oral dosage forms using highperformance liquid chromatography with evaporative light-scattering detection. J Pharm Biomed Anal, 41:731-737, 2006.

[25]. V D P. Nair and I. Kanfer. High-Performance Liquid Chromatographic Method for the Quantitative Determination of Hypoxoside in African Potato (Hypoxis hemerocallidea) and in Commercial Products Containing the Plant Material and/or its Extracts. J Agric Food Chem, 54:2816-2821, 2006.

[26]. V D P. Nair and I. Kanfer. A Capillary Zone Electrophoresis (CZE) Method for the Quantitative Determination of Hypoxoside in Commercial Formulations of African Potato 
(Hypoxis hemerocallidea). Phytochem Anal, 18:475-483, 2007.

[27]. Guidance for industry- dissolution testing of immediate release solid oral dosage forms. U.S. Department of Health and Human Services, Food and Drug Administration (FDA), Center for Drug Evaluation and Research (CDER), Rockville, U.S.A. 1997.

[28]. USP-NF Product Information. General Information-Nutritional Supplements. United States Pharmacopoeial Convention, Rockville, U.S.A., pp 2663-2670, 2005.

[29]. K. Westerhoff, A. Kauzinger, M. Wurglics, J. Dressman and M. Schubert-Zsilavecz. Biorelevant dissolution testing of St. John's wort products. J Pharm Pharmacol, 54:16151621, 2002.

[30]. M. Ganzera, J. Zhao and I A. Khan. Hypericum perforatum- Chemical profiling and quantitative results of St. John's wort products by an improved high performance liquid chromatography method. J Pharm Sci, 91:623630, 2002.

[31]. Note for the guidance on the investigation of bioavailability and bioequivalence (CPMP/EWP/QWP/1401/98), committee for propriety medicinal products (CPMP). The European Agency for the Evaluation of Medicinal Products (EMEA) London, U.K., 2001.

[32]. E. Galia, E. Nicolaides, D. Horter, R. Lobenberg, C. Reppas and J B. Dressman. Evaluation of various dissolution media for predicting in vivo performance of class I and class II drugs. Pharm Res, 15:698-705, 1998.

[33]. J. Dressman and C. Reppas. In vitro-in vivo correlations for lipophilic, poorly water-soluble drugs. Eur J Pharm Sci, 11:S73-S80, 2000.

[34]. R. Ostlund and W. Sherman. Pinotol and derivatives thereof for the treatment of metabolic disorders. U.S. Patent 5,882,896, 1996.

[35]. R. Ostlund, C. Spilburg and W. Steson. Sitostanol administered in lecithin micelles potently reduces cholesterol absorption in humans. Am J Clinic Nutr, 70:826-831, 1999. 